\title{
B-cell Lymphoma Detected by a Pulse Oximeter
}

\author{
Arine Musaelyan, MD' - Aaron Park, MD' - Ruxing Xing, MD' - Ritu Chakrabarti, MD' - Xinlai Sun, MD2 - Stefan Balan, MD $^{3}$
}

A 60-year-old man with no significant medical or smoking history presented to our emergency department (ED) after detecting persistently low $\mathrm{SpO} 2$ measurements of $89 \%$ to $91 \%$ on his home pulse oximeter device during the peak of the COVID-19 pandemic.

The patient worked as a physician and denied dyspnea, fever, cough, and chest pain. His family history was noncontributory.

Upon arrival, the patient was hypoxic with an oxygen saturation level of $91 \%$ on room air. The physical examination was unremarkable, with no lymphadenopathy noted. Initial laboratory results were significant only for mild leukocytosis with a white blood cell count of $11 \times 109 / \mathrm{L}$ (reference range, 4.5-11.0 $\times 109 / \mathrm{L}$ ). The differential included neutrophils, $87 \%$; lymphocytes, $7.7 \%$; and monocytes, $4.7 \%$.

\section{Diagnostic testing}

Given the patient's hypoxia, a computed tomography (CT) angiography of the chest was conducted. The results were negative for pulmonary embolism but
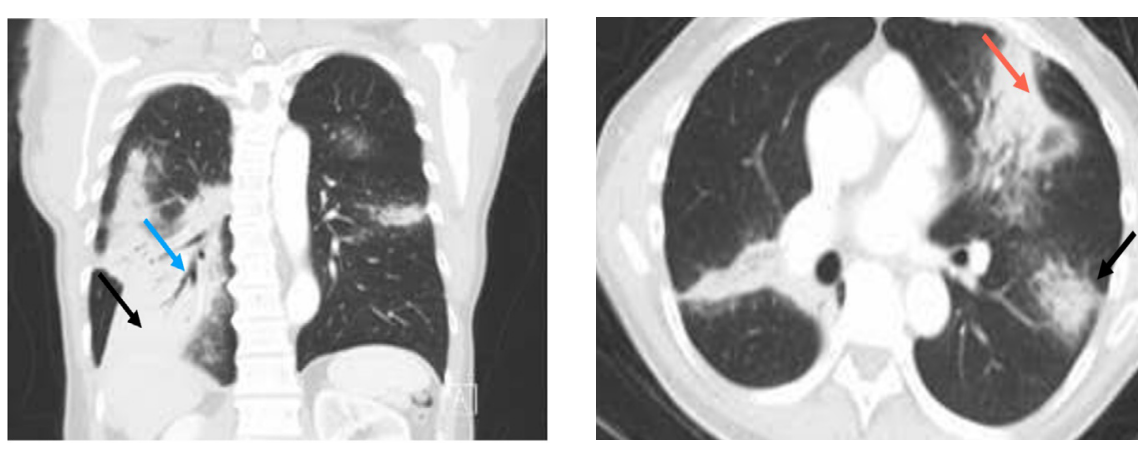

Figure 1. Coronal ( $A$, left) and transverse (B, right) views showed dense consolidations of the right lower lobe (black arrow) with bronchograms (blue arrow), inferior left upper lobe, lingular, and superior segment of the left upper lung (red arrow), as well as scattered tree-in-bud nodularity and areas of ground glass opacities. revealed possible multifocal pneumonia predominantly in the bilateral lower lobes. The patient was initially treated with hydroxychloroquine, azithromycin, and amoxicillin clavulanate for community-acquired pneumonia. Two polymerase chain reaction (PCR) tests for COVID-19 returned negative results, and the patient was discharged home.

Despite the completion of the antibiotic course, the patient returned to the ED with

\section{AFFILIATIONS:}

'Resident, Internal Medicine Department, Jersey City Medical Center, Jersey City, New Jersey

${ }^{2}$ Director and Chair, Department of Pathology and Laboratory Medicine, Jersey City Medical Center, Jersey City, New Jersey

${ }^{3}$ Chief, Oncology Department, Jersey City Medical Center, Jersey City, New Jersey

\section{CITATION:}

Musaelyan A, Park A, Xing R, Chakrabarti R, Sun X, Balan S. B-cell lymphoma detected by a pulse oximeter. Consultant. Published online May 28, 2021. doi:10.25270/con.2021.05.00014

Received January 12, 2021. Accepted February 19, 2021.

\section{DISCLOSURES:}

The authors report no relevant financial relationships.

\section{CORRESPONDENCE:}

Arine Musaelyan, MD, Jersey City Medical Center, 355 Grand Street, Jersey City, NJ, 07302 (arine.md@ gmail.com) persistent hypoxemia. Repeat CT of the chest showed unchanged dense consolidations of the right lower lobe, inferior left upper lobe, lingula, and superior segment of the left upper lung, with internal air bronchograms as well as scattered tree-inbud nodularity and areas of ground glass opacities (Figures 1a and 1b).

Positron emission tomography (PET) and CT scans revealed low-grade activity with standardized uptake value (SUV) of up to 2.9 and anatomic and metabolic patterns suggestive of chronic indolent infection, although the underlying malignancy could not be excluded (Figures 2a and $2 b$ ). Diagnostic bronchoscopy with biopsy was performed.

A histopathologic examination revealed sheets of small blue cell infiltration. Morphologically, these small blue cells had the appearance of mature lymphocytes (Figure 3a), which were confirmed to be B-cells by immunohistochemical staining. The cells were positive for CD45, CD20 (Figure 3B), and $\mathrm{Bcl}-2$ and were negative for CD5, CD10, CD43, Bcl-1, AE1/ 

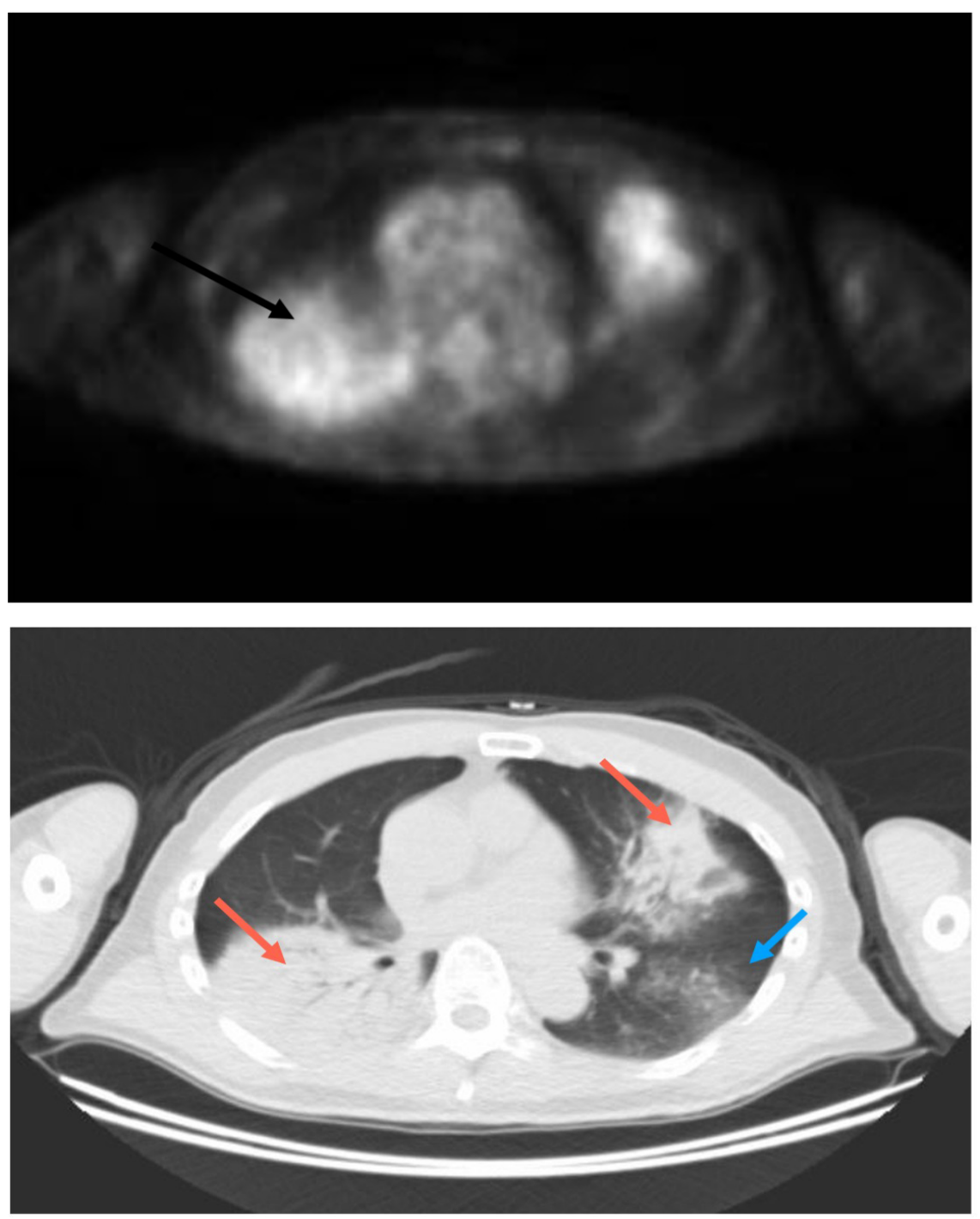

Figure 2. A PET scan (A, top) illustrated the areas of dense consolidation with low-grade activity with maximum SUV of approximately 2.9, suggestive of chronic infection vs malignancy (black arrows). A CT scan (B, bottom) revealed right lower and left upper lobe consolidations (red arrows) and ground glass opacities (blue arrow).

AE3, chromogranin, and synaptophysin. Immunostain for Ki-67 showed a low proliferation rate of $2 \%$ to $3 \%$. A PCR test for $B$-cell immunoglobulin $\mathrm{H}$ gene rearrangement was performed using the paraffin embedded tissue, results of which were positive (a clonal B-cell gene rearrangement was detected).

Therefore, a low-grade B-cell lymphoma consistent with a marginal zone lymphoma was diagnosed, and the patient was initiated on bendamustine plus rituximab therapy.

\section{Discussion}

As of May 2021, more than 164 million cases of COVID-19 have been confirmed worldwide! COVID-19 mortality rates in some countries were as high as $15 \%$ throughout the pandemic.! This unprecedented global health pandemic not only reorganized the economy but also changed the way people live on a day-to-day basis, including the items they purchase.

Pulse oximeters went out of stock in the largest retailers when people learned that a low oxygen saturation level could be a sign of possible COVID-19 infection. ${ }^{2}$ The typical cost of these devices ranges from $\$ 25$ to $\$ 100$, and data show that US sales of pulse oximeters increased by $506 \%$ during the week of February $17,2020 .{ }^{2}$ Since then, demand has held steady. In mid-May 2020, the devices were still sold out, delayed, or on backorder in major retail stores. ${ }^{3-5}$

The question persists: Are these nonmedical, commercial pulse oximeters necessary or even reliable tools for the general population? ${ }^{3}$ The American Lung Association advises against purchasing a pulse oximeter and to rather focus on early awareness of COVID-19 symptoms, such as cough, shortness of breath, chest discomfort, respiratory rate, and pulse rate. ${ }^{5}$ The organization argues that unless a preexisting chronic lung or heart condition exists that could affect baseline oxygen levels, most individuals do not require a home pulse oximeter. ${ }^{5}$

On the other hand, several cases have been reported in which patients with COVID-19 do not display symptoms but incidentally display low oxygen levels through pulse oximetry readings. Called "silent hypoxia," hypoxemia for these patients tends to be out of proportion to their respiratory effort. An argument has been made for these patients to closely monitor their oxygen levels with pulse oximetry for early recognition of possible decompensation and to identify the need to return to the hospital for further evaluation. ${ }^{6}$

Overall, shared concerns have been raised regarding erroneous pulse-oximetry readings, the variability of performance among different manufacturers' devices, and incorrect usage. Such inconsistencies may prompt unnecessary care and false reassurances with normal oxygen saturation level in patients with COVID-19., Ironically in our case, a physician's purchase of a pulse oximeter led to a life-changing discovery of a rare malignancy.

Non-Hodgkin lymphoma is known to originate from lymphocytes and can arise in multiple organs and systems, including 

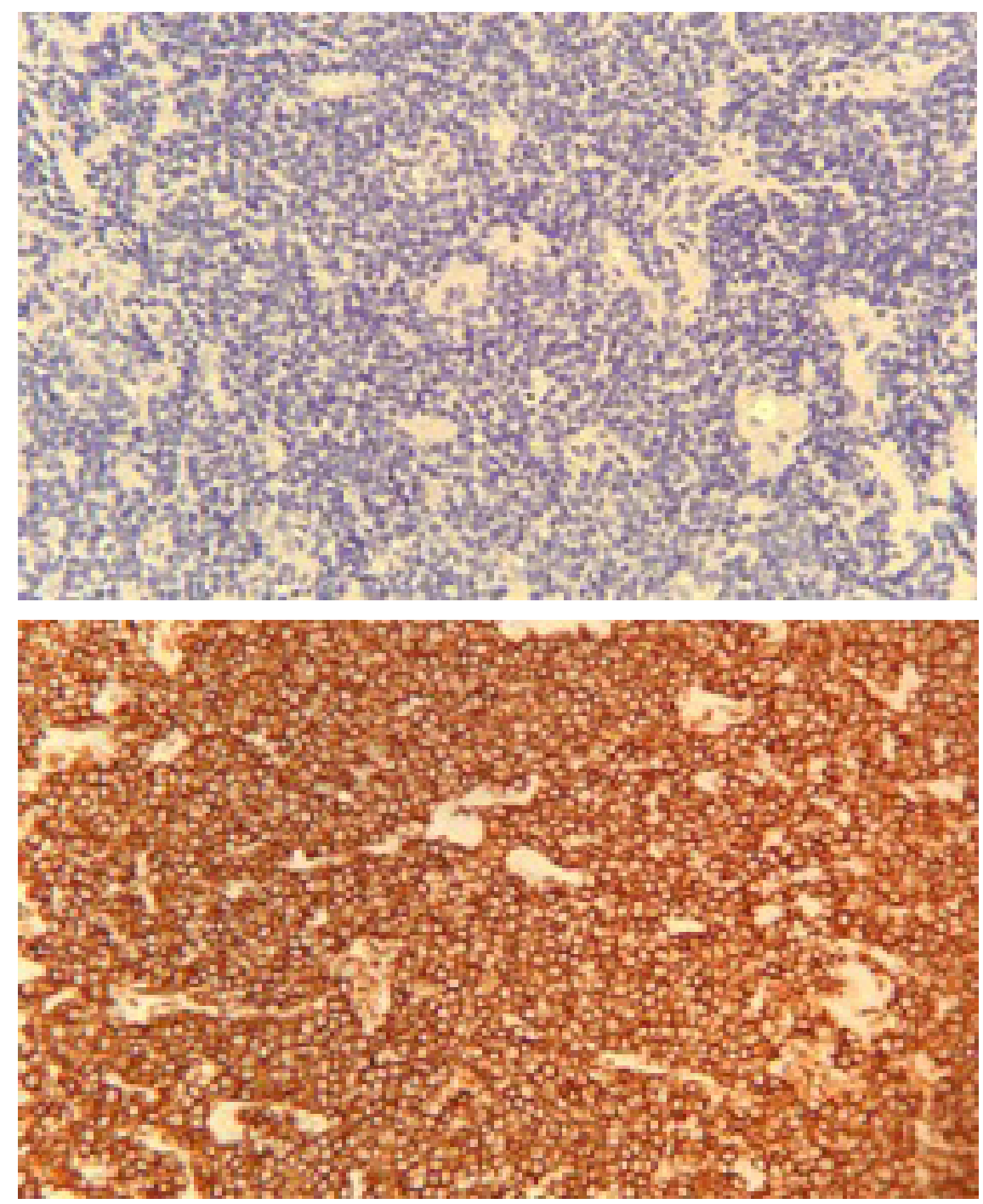

Figure 3. A histopathologic examination revealed small blue cells with the appearance of mature lymphocyes in the transbronchial lung biopsy (A, top, 400x). A immunohistochemical stain confirmed these findings, results of which were positive for CD45 and CD20 (B, bottom, 400x).

the stomach, bowel, thyroid, lung, salivary glands, ocular adnexa, and skin. ${ }^{8,9}$ In the 2017 World Health Organization classification, extranodal marginal zone lymphoma of mucosa-associated lymphoid tissue (MALT) accounted for $7 \%$ to $8 \%$ of all B-cell lymphomas, of which $9 \%$ represented a formerly called bronchial-associated lymphoid tissue (BALT) lymphoma. ${ }^{10}$ Pulmonary MALT lymphoma arises from bronchus-associated lymphoid tissue and comprises small lymphocytes with focal plasmacytoid features. ${ }^{11}$ It is usually seen in patients aged older than 60 years, and its prognosis is favorable with a 5 -year survival rate of more than $85 \%$ and a median survival rate of more than 10 years in the largest reported series. ${ }^{10}$

Certain conditions are found to be associated with pulmonary lymphoid hyperplasia, such as preexisting follicular bronchiolitis, chronic antigenic stimulation from smoking, autoimmune inflammatory processes, and acute infections. ${ }^{12}$ While Helicobacter pylori has been known for the strong pathophysiological association with gastrointestinal MALT lymphoma, there is no common antigen responsible for the development of the majority of pulmonary MALT lymphomas. ${ }^{12}$ The association of rheumatoid arthritis, systemic lupus erythematosus, or Sjörgen syndrome with the increased risk of developing non-Hodgkin lymphoma has been described.13

The most common presenting symptoms of pulmonary MALT lympthoma are cough, chest pain, and dyspnea, but 55\% of patients are found to be asymptomatic upon initial diagnosis..$^{2}$ Imaging studies are commonly suggestive of one or multiple lung nodules, masses, areas of air space consolidation, air bronchograms, ground glass opacity, tree-in-bud sign, or reticular lesions. About $60 \%$ to $70 \%$ of patients may present with multifocal findings. ${ }^{14}$

Different treatment options have been available for the management of pulmonary MALT lymphoma, which can include surgery and radiation for localized disease and chemotherapy or immunotherapy for diffuse disease..$^{15}$ Based on the results of the BRIGHT study, bendamustine plus rituximab is the preferred regimen over R-CHOP (rituximab plus cyclophosphamide, doxorubicin, vincristine, and prednisone). While evaluation of overall survival showed no significant difference between the study groups, it was concluded that treatment with bendamustine plus rituximab provides greater disease control compared with R-CHOP/R-CVP (cyclophosphamide, vincristine, prednisone) treatment. ${ }^{16}$

\section{Conclusion}

The novel coronavirus pandemic sparked a sudden interest in home pulse oximeters. This noninvasive tool that measures arterial blood oxygen saturation represents an accessible tool that can be easily used by patients and health care providers. However, there are mixed opinions regarding its use. While some proponents argue that these devices could provide early recognition of hypoxia and therefore treatment, other providers still question its overall benefit for asymptomatic individuals. In our case, purchase 
of a pulse oximeter helped screen for and eventually unmask a potentially harmful diagnosis had it been left untreated. Overall, we can conclude that routine home use of pulse oximeters continues to be indicated for patients with COVID-19 who have preexisting chronic lung or heart conditions and those who have a high risk of disease progression.

\section{References}

1. WHO Coronavirus (COVID-19) Dashboard. World Health Organization. Accessed May 18, 2021. https://covid19.who.int/

2. Daily $L$. What is a pulse oximeter, and does the coronavirus pandemic mean you need one? Washington Post. Published May 19, 2020. Accessed May 4, 2021. https://www.washingtonpost. com/lifestyle/wellness/pulse-oximeter-covid-19-coronavirus/2020/05/18/5b6f8a98-96df-11ea-9f5e-56d8239bf9ad_story.html

3. Peachman RR. People concerned about COVID-19 are using pulse oximeters to measure oxygen levels. These are the pros and cons. Consumer Reports. Published April 10, 2020. Accessed May 4, 2021. https://www.consumerreports.org/ medical-symptoms/covid-19-pulse-oximeters-oxygen-levels-faq

4. Khalid A. Pulse oximeters are selling out because of the pandemic. Quartz. Published April 4, 2020. Accessed May 4, 2021. https://qz.com/1832464/pulse-oximeters-for-coronavirus-unnecessary-but-selling-strong

5. Pulse oximeter not a substitute for talking to healthcare provider, watching for early COVID-19 symptoms. News release. American Lung Association; April 30, 2020. Accessed May 4, 2021. https://www.lung. org/media/press-releases/pulse-oximeter-covid-19

6. Quaresima V, Ferrari M. COVID-19: efficacy of prehospital pulse oximetry for early detection of silent hypoxemia. Crit Care. 2020;24(1):501-502. https://doi.org/10.1186/ s13054-020-03185-x

7. Parker-Pope T. What's a pulse oximeter, and do I really need one at home? New York Times. Published April 24, 2020. Accessed May 4, 2021. https://www.nytimes. com/2020/04/24/well/live/coronavirus-pulse-oximeter-oxygen.html

8. Lee I-J, Kim SH, Koo SH, et al. Bronchus-associated lymphoid tissue (BALT) lymphoma of the lung showing mosaic pattern of inhomogeneous attenuation on thin-section CT. Korean J Radiol. 2000;1(3):159-161. https:// doi.org/10.3348/kjr.2000.1.3.159

9. Ferraro $P$, Trastek VF, Adlakha H, Deschamps C, Allen MS, Pairolero PC. Primary non-Hodgkin's lymphoma of the lung. Ann Thoracic Surg. 2000;69(4):993-997. https:// doi.org/10.1016/S0003-4975(99)01535-0

10. Cook JR. Extranodal marginal zone lymphoma of mucosa-associated lymphoid tissue (MALT lymphoma). In: Swerdlow $\mathrm{SH}$, Campo E, Harris NL, et al, eds. WHO Classification of Tumours of Haematopoietic and Lymphoid Tissues. 4th ed, vol 2. International Agency for Research on Cancer; 2017:259-262.

11. Chow WH, Ducheine Y, Hilfer J, Brandstetter RD. Chronic pneumonia: Primary malignant
non-Hodgkin's lymphoma of the lung arising in mucosa-associated lymphoid tissue. Chest. 1996;110(3):838-840. https://doi. org/10.1378/chest.110.3.838

12. Sammassimo S, Pruneri G, Andreola G, et al. A retrospective international study on primary extranodal marginal zone lymphoma of the lung (BALT lymphoma) on behalf of International Extranodal Lymphoma Study Group (IELSG). Hematol Oncol. 2016;34(4):177-183. https://doi.org/10.1002/ hon.2243

13. Borie $\mathrm{R}$, Wislez $\mathrm{M}$, Thabut $\mathrm{G}$, et al. Clinical characteristics and prognostic factors of pulmonary MALT lymphoma. Eur Resp J. 2009;34(6):1408-1416. https://doi. org/10.1183/09031936.00039309

14. King LJ, Padley SPG, Wotherspoon AC, Nicholson AG. Pulmonary MALT lymphoma: imaging findings in 24 cases. Eur Radiol. 2000;10:1932-1938. https://doi.org/10.1007/ s003300000491

15. Beal KP, Portlock C, Yahalom J. Bronchial-Associated lymphoid tissue (BALT) lymphoma: characteristics and treatment outcome of 19 cases. Blood. 2004;104(11):4564. https://doi.org/10.1182/ blood.V104.11.4564.4564

16. Flinn IW, van der Jagt $R$, Kahl B, et al. First line treatment of patients with indolent non-Hodgkin lymphoma or mantle-cell lymphoma with bendamustine plus rituximab versus R-CHOP and R-CVP: Results of the BRIGHT 5-year Follow-Up Study. J Clin Oncol. 2019;37(12):984-991. https://doi. org/10.1200/JCO.18.00605 